\title{
Manifestation of Pleomorphic Undifferentiated Aortic Sarcoma with Splenic Infarction: A Case Report
}

\author{
Varun Kaushala Behzad Amoozgar ${ }^{a}$ b Nabonita Barua ${ }^{a}$ \\ Shankar Chhetria Bhaveshkumar Garsondiya ${ }^{a}$ \\ aDepartment of Internal Medicine, Jersey Shore University Medical Center, Perth Amboy \\ Division, Perth Amboy, NJ, USA; bUniversity of California, Berkeley, School of Public Health, \\ Berkeley, CA, USA
}

\section{Keywords}

Aortic sarcoma - Undifferentiated pleomorphic sarcoma - Splenic infarction · Magnetic resonance imaging

\begin{abstract}
Aortic spindle cell sarcoma is a rare neoplasm with poor prognosis that is often found incidentally due to its adverse effects. CT and MRI with contrast are useful imaging modalities, but a tissue biopsy is the gold standard for diagnosis. Tumor resection is the ultimate treatment followed by chemotherapy. Our case was an adult female who presented mainly for shortness of breath, and further imaging workup demonstrated a soft tumor juxtaposed to a major vein with compressive effect. The patient's tumor was resected, and the pathology result confirmed undifferentiated aortic sarcoma. The patient's condition improved and she was discharged with outpatient oncology follow-up and possible treatment.
\end{abstract}

(C) 2020 The Author(s).

Published by S. Karger AG, Basel

\section{Introduction}

The spectrum of sarcomas is broad and it has the capacity to arise and mature into smooth muscle, skeletal muscle, adipose, fibrous tissue, and bone. Large artery primary sarcomas represent less than $1 \%$ of all sarcomas [1]. Tumors grow at various rates depending on the aggressiveness of the tumor and often compress nearby structures. High-grade sarcomas have the propensity to metastasize hematogenously and cause acute arterial embolisms [2]. They tend to arise in the 5th-7th generation of life and occur more often in men. Diagnosis of 
Fig. 1. Area of soft tissue (red arrow) surrounding the margins of the ascending thoracic aorta and narrowing the superior vena cava (green arrow).

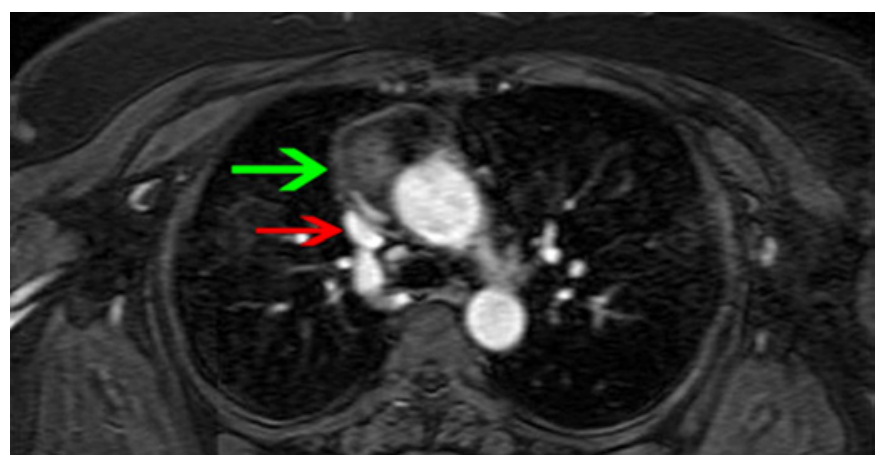

soft tissue sarcomas is often unsuspected and if inappropriate excision is done before proper pathological diagnosis, they have a tendency for recurrence, metastasis, and requirements of a larger procedure to be done subsequently [3]. Here, we present a case of a 47-year-old female with high-grade undifferentiated pleomorphic sarcoma of the ascending aorta manifested primarily as splenic infarction. The tumor was only better visualized on magnetic resonance imaging (MRI) and ultimately due to its location required complete excision for an official diagnosis.

\section{Case Presentation}

A 47-year-old female with a past medical history of hypertension and fibromyalgia presented to ER for shortness of breath (SOB) for 2 days; There was a sudden onset of SOB that was worsening with exertion and was associated with non-radiating upper mid-back pain, dizziness, and chest pain. The patient also endorsed 2 weeks of dark stools and diarrhea. The patient's initial vitals were BP: $165 / 94$, HR: $116 / \mathrm{min}, \mathrm{T}: 98.3^{\circ} \mathrm{F}$, and oxygen saturation of $100 \%$ on room air. On the physical exam, the patient had pallor and was stool guaiac positive. Her labs showed a WBC: $7.4 \times 10^{3} / \mathrm{mL}, \mathrm{Hg}: 5.8 \mathrm{~g} / \mathrm{dL}, \mathrm{BUN}: 7 \mathrm{mg} / \mathrm{dL}, \mathrm{Cr}: 0.7 \mathrm{mg} / \mathrm{dL}$, and D-dimer of $0.94 \mathrm{mg} / \mathrm{L}$.

Computerized tomography angiography (CTA) of abdomen and pelvis was initially done to explore the etiology for gastrointestinal bleeding, which showed colonic diverticulosis and a wedge-shaped hypoattenuation involving the spleen, anteriorly and inferiorly, which was suggestive of a splenic infarct. The patient was admitted to ICU for GI bleeding and an appropriate bleeding protocol was initiated. The patient was transfused 1 unit of PRBC. The patient had esophagogastroduodenoscopy the following morning, which found mild gastritis and duodenitis. Computerized tomography of the chest with contrast was performed to rule out PE, which found low attenuation collection within the mediastinum, centered upon the ascending aorta, consistent with possible hematoma secondary to remote trauma. Consecutively, MRI of the chest and abdomen was ordered. MRI of the abdomen still could not rule out splenic infarct and was highly suggestive of it as it showed a somewhat triangular shape with no internal enhancement. Furthermore, MRI chest showed a soft tissue mass associated with the ascending thoracic aorta, with a mass effect on the superior vena cava with about $90 \%$ narrowing in the inferior aspect as it approaches the right atrium (Fig. 1).

Thoracic surgery and Hematology-oncology opted to transfer the patient to a tertiary facility for cardio-thoracic evaluation. There she first underwent cardiac catheterization, which showed mild luminal irregularities in the middle of the left anterior descending artery and distal right coronary artery. The patient went to the operating room, in which the excision of tumor en masse and replacement of ascending aorta (hemiarch) under deep hypothermic circulatory arrest were done. Preliminary pathology reports at that time were showing spindle cell neoplasm of the aorta and Hematology-oncology was consulted. On post-op day

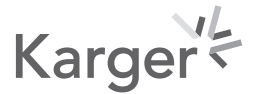


two, the patient developed right pneumothorax and a pigtail catheter was placed and subsequently removed on post-op day five.

The patient was safely discharged home and to follow-up with oncology for final path results and treatments. Final pathology reports showed high-grade spindle and pleomorphic sarcoma (undifferentiated pleomorphic sarcoma). Pathologist further sent the sample to the Sloan Kettering, which showed that there was a lack of CDK4 and MDM 2 expression, arguing against intimal sarcoma.

\section{Discussion}

Primary aortic sarcoma is a rare disease and difficult to diagnose perioperatively. There are less than 200 reported cases within the literature. These tumors more often occur in men compared to women and the mean age of occurrence is about 60 years old [4]. The prognosis for these tumors remains poor, with the median age of survival about 11 months [4]. Primary aortic sarcoma is a rare disease entity and most commonly occurs in the ascending aorta or aortic arch (11\%) in comparison to the descending aorta, where it occurs more frequently [5]. No definitive risk factors have been found for primary malignant tumors of the aorta, but some studies have postulated that one possible risk factor is from implantation of Dacron vascular grafts or other foreign bodies that contain carcinogens [6]. It is still unclear if these findings are more coincidental as this disease is so rare.

Aortic sarcomas often present with signs of vascular diseases including embolic events and aneurysm. The presenting symptoms may be abdominal pain, SOB, chest pain, nausea, and vomiting [7]. Both SOB and clinical signs of an embolic event occurred in this patient.

Aortic sarcomas can arise from the adventitia, media, or the intima. Intimal lesions are the most common and often manifest with embolic events or obstruction of the aorta and or surrounding vessels [7]. Tumors of the media or adventitia cause a more mass-like effect. The aortic arch sarcoma, which is intimal in origin, is associated with cerebral emboli and early metastasis to the brain. Vascular symptoms occur when the tumor compresses the lumen or invades the surrounding vessels such as the vena cava, as seen in this case.

The rarity and location of this disease are often found incidentally. In addition, it is difficult to distinguish these malignancies from other vascular disorders such as inflammatory aortic diseases due to similar radiological features. Best detecting modalities helping in differentiating this disease from other disorders are CTA, transesophageal echocardiogram, MRI, and positron emission tomography scans [8]. In our patient, detection was done via CTA chest and MRI for the primary site of the tumor, guided by splenic infarction possibly due to an embolic event. Hence, imaging tools are useful in locating the tumor and place it higher on the differentials. However, the gold standard for diagnosis is still biopsy and pathology.

By the time of diagnosis, metastasis already occurred in $80 \%$ of the patients [9]. Tumor emboli mostly involve liver, lungs, and bones [10]. Arterial embolization occurs in $46.7 \%$ of diagnosed patients [11]. The likelihood of an embolic or metastatic event occurring in spindle cell sarcoma appears to be related to the anatomic site of origin, which allows direct vascular access and procoagulant state. This is similar in this case, in which splenic embolization and infarction had occurred by the time of diagnosis. Questions regarding prophylactic therapy in patients with undifferentiated pleomorphic sarcoma have been suggested pertaining to high embolic rates.

Surgical resection remains the primary treatment option and gives the best chance for cure and consecutively reduction in mortality. Due to the latency in diagnosis and early metastasis, the overall prognosis is poor for these patients [11]. Complete resection still appears to be the best option as it offers a prolonged survival [12]. 


\section{Conclusion}

Herein we report a rare case of aortic sarcoma, associated with splenic infarction. Although treatment of this tumor is challenging due to its aggressive nature and high rate of metastatic and induced embolic events, complete resection will help promote higher survival rate.

\section{Statement of Ethics}

Written informed consent was obtained from the patient for publication purposes.

\section{Conflict of Interest Statement}

The authors declare that there is no conflict of interest regarding the publication of this paper. The authors confirm that no organization funded this study. The authors are the only people who have made substantive contributions to the study.

\section{Funding Sources}

The authors acknowledge that they did not receive any funding for this study.

\section{Author Contributions}

Varun Kaushal, MD: data collection, preparation, and revision of the manuscript. Behzad Amoozgar, MD: revision of the manuscript. Nabonita Barua, MD: revision of the manuscript. Shankar Chhetri, MD: preparation and revision of the manuscript. Bhaveshkumar Garsondiya, MD: preparation and revision of the manuscript.

\section{References}

1 Von Falck C, Meyer B, Fegbeutel C, Länger F, Bengel F, Wacker F, et al. Imaging features of primary sarcomas of the great vessels in CT, MRI and PET/CT: a single-center experience. BMC Med Imaging. 2013; 13(1): 25.

2 Rusthoven CG, Liu AK, Bui MM, Schefter TE, Elias AD, Lu X, et al. Sarcomas of the aorta: a systematic review and pooled analysis of published reports. Ann Vasc Surg. 2014; 28(2): 515-25.

3 Rehders A, Stoecklein NH, Poremba C, Alexander A, Knoefel WT, Peiper M. Reexcision of soft tissue sarcoma: sufficient local control but increased rate of metastasis. World J Surg. 2009; 33(12): 2599-605.

4 Rosado-de-Christenson ML, Carter BW. Specialty Imaging: Thoracic Neoplasms E-Book. Elsevier Health Sciences. 2015.

5 Chiche L, Mongrédien B, Brocheriou I, Kieffer E. Primary tumors of the thoracoabdominal aorta: surgical treatment of 5 patients and review of the literature. Ann Vasc Surg. 2003; 17(4): 354-64.

6 Dietl CA, Samedi VG, Langsfeld M. Malignant aortic tumors following open and endovascular implantation of prosthetic vascular grafts. Cardiovascular Thoracic Surgery 2018; 3(4): 1-7.

7 Espada R, Iliopoulos D, Weilbaecher D. Sarcoma presenting as thoracoabdominal aortic aneurysm: case reports and literature review. Contemp Surg. 1966; 48: 135-9.

8 Wu ZY, Weng LQ, Chen ZG, Chen YX, Li YJ. Primary aortic sarcoma in arch and descending aorta: a case report and literature review. J Thorac Dis. 2018; 10(4): E289.

9 Ibrahim A, Luk A, Singhal P, Wan B, Zavodni A, Cusimano RJ, et al. Primary intimal (spindle cell) sarcoma of the heart: a case report and review of the literature. Case Rep Med. 2013; 2013: 461815.

10 Rusthoven C, Shames ML, Bui MM, Gonzalez RJ. High-grade undifferentiated pleomorphic sarcoma of the aortic arch: a case of endovascular therapy for embolic prophylaxis and review of the literature. Vasc Endovascular Surg. 2010; 44(5): 385-91.

11 Burke AP, Virmani R. Sarcomas of the great vessels. A clinicopathologic study. Cancer. 1993; 71(5): 1761-73.

12 Fatima J, Duncan AA, Maleszewski JJ, Kalra M, Oderich GS, Gloviczki P, et al. Primary angiosarcoma of the aorta, great vessels, and the heart. J Vasc Surg. 2013; 57(3): 756-64. 